\title{
Morphometric differences in planum temporale in schizophrenia and bipolar disorder revealed by statistical analysis of labeled cortical depth maps
}

\section{J. Tilak Ratnanather ${ }^{1,2,3}{ }^{*}$, Shannon Cebron ${ }^{1}$, Elvan Ceyhan ${ }^{4}$, Elizabeth Postell ${ }^{1}$, Dominic V. Pisano ${ }^{1}$, Clare B. Poynton ${ }^{1}$, Britni Crocker ${ }^{1}$, Nancy A. Honeycutt ${ }^{5}$, Pamela B. Mahon ${ }^{5}$ and Patrick E. Barta ${ }^{1,2}$}

${ }^{1}$ Center for Imaging Science, Johns Hopkins University, Baltimore, MD, USA

2 Institute for Computational Medicine, Johns Hopkins University, Baltimore, MD, USA

${ }^{3}$ Department of Biomedical Engineering, Johns Hopkins University, Baltimore, MD, USA

${ }^{4}$ Department of Mathematics, Koç University, Istanbul, Turkey

${ }^{5}$ Department of Psychiatry, Johns Hopkins University School of Medicine, Baltimore, MD, USA

\section{Edited by:}

Bernat Kocsis, Harvard Medical

School, USA

\section{Reviewed by:}

Bernhard J. Mitterauer, Volitronics-Institute for Basic

Research Psychopathology and Brain Philosophy, Austria

Nicolas Bolo, Beth Israel Deaconess

Medical Center, USA

*Correspondence:

J. Tilak Ratnanather, Center for

Imaging Science, The Johns Hopkins University, Clark 301, 3400 North

Charles Street, Baltimore, MD 21218, USA

e-mail: tilak@cis.jhu.edu
Differences in cortical thickness in the lateral temporal lobe, including the planum temporale (PT), have been reported in MRI studies of schizophrenia (SCZ) and bipolar disorder (BPD) patients. Most of these studies have used a single-valued global or local measure for thickness. However, additional and complementary information can be obtained by generating labeled cortical distance maps (LCDMs), which are distances of labeled gray matter (GM) voxels from the nearest point on the GM/white matter (WM) (inner) cortical surface. Statistical analyses of pooled and censored LCDM distances reveal subtle differences in PT between SCZ and BPD groups from data generated by Ratnanather et al. (Schizophrenia Research, http://dx.doi.org/10.1016/j.schres.2013.08.014). These results confirm that the left planum temporale (LPT) is more sensitive than the right PT in distinguishing between SCZ, BPD, and healthy controls. Also confirmed is a strong gender effect, with a thicker PT seen in males than in females. The differences between groups at smaller distances in the LPT revealed by pooled and censored LCDM analysis suggest that SCZ and BPD have different effects on the cortical mantle close to the GMMM surface. This is consistent with reported subtle changes in the cortical mantle observed in post-mortem studies.

\section{INTRODUCTION}

Recent studies suggest that thicknesses of cortical structures seen in MRI scans may be different in patients with neurodevelopmental diseases, such as schizophrenia (SCZ) and psychotic bipolar disorder (BPD), and that these changes may be related to genetic and environmental factors (1-6). In particular, differences in cortical thickness have been associated with abnormalities in the posterior or caudal superior temporal gyrus (STG), part of which includes the planum temporale (PT). The PT is a multisensory region on the ventral surface of the STG and plays an important role in language and speech processing (7). These functions may be disrupted by auditory hallucinations resulting in the classic psychotic symptoms of SCZ (8), which differ from those seen in BPD (9). Thus, the PT continues to be important in neuroimaging studies of SCZ.

One method of quantifying cortical thickness is labeled cortical distance mapping (LCDM), which analyzes a subvolume encompassing the region of interest (ROI). The approach requires precise definitions of anatomical boundaries, which can be compounded by interindividual variability. The boundary between white matter (WM) and CSF is often indistinct, but this challenge can be partially overcome by viewing the ROI as a laminar structure composed of gray matter (GM) voxels and a local surface coordinate system based on an anatomically defined GM/WM cortical surface (10). This approach is in line with the classic formulation of differential geometry, in which local coordinate systems are examined. In this case, the LCDM data are represented by a set of distances from labeled GM voxels with respect to the nearest point on the GM/WM surface.

There are several ways of analyzing LCDM data. The simplest is to use the 95th percentile of the LCDM as the overall thickness, a definition, which has been used in several neuroimaging studies of SCZ in the cingulate $(11,12)$, prefrontal cortex (13), and parahippocampal gyrus (14). A second approach is Local LCDM, which condenses to a single distance value at each point on the surface. Local LCDM was used to examine variation in thickness across cingulate (15) and the left PT (16) surfaces in SCZ. In the latter study, the variation in thickness was found to be consistent with post-mortem analysis (17). Finally, a third approach is to define a "laminar thickness" as the number of voxels at the 95th percentile LCDM, divided by surface area. This was used in an expansion of the Qiu et al. (16) study and revealed gender and laterality effects on the PT, with reduced laterality found in SCZ but not BPD (18). Approaches similar to LCDMs have also been developed and used in other studies (19-26). 
This paper describes the use of a fourth approach, namely pooled and censored LCDM analysis, that can offer further information than just summary measures of thickness and volume. For example, it is possible to pool the LCDM distances for each diagnostic group and perform statistical comparisons on the overall distance level for each group, rather than the individual level; further, it is possible to censor these distances to compare the diagnostic groups at specific distances from the GM/WM surface $(27,28)$. This is achieved by merging the distances for subjects from the same group to utilize more of the information provided in these distances. The other three methods use summary measures based on LCDM distances and tend to over summarize the information contained in these distances. Hence, group differences in tissue morphometry might be missed. In this fourth method of pooled and censored LCDMs, the common group morphometric traits are emphasized and the effect of individual variation is lessened. For example, pooling appears to be effective even with small to medium sample sizes (i.e., number of cases and controls) in structures known to be affected by disease such as the cingulate in dementia (29) and deficit SCZ (30). Thus, we aim to demonstrate that applying the pooled and censored LCDM technique to the Ratnanather et al. (18) dataset offers an alternative and interesting way of quantifying differences in the effect of SCZ and BPD within the PT cortical mantle.

\section{METHODS \\ DATA}

Details of subjects, MRI acquisition, segmentation, and GM/WM surface reconstruction are described in Ratnanather et al. (18). A brief description follows. Using a 1.5-T scanner, T1 scans with $1 \mathrm{~cm}^{3}$ isotropic resolution across the entire cranium were obtained for 94 subjects grouped on age, sex, and ICV. Study groups included healthy controls $[n=27,39.9 \pm 11.1$ (mean $\pm \mathrm{SD}$ ) years old, $52.8 \%$ male], SCZ ( $n=31,41.4 \pm 9.5$ years old, $54.8 \%$ males), and BPD ( $n=36,44 \pm 15.6$ years old, $44.4 \%$ males). Bayesian segmentation of ROI masks for the STG was used to classify image voxels as GM, WM, or CSF. The GM/WM threshold from the segmentation was used to generate triangulated isosurfaces representing the $2 \mathrm{D}$ cortical surface. The subsurface corresponding to the PT was extracted by cutting the triangulated surface via curvature-based dynamic programing delineation of gyral and sulcal boundaries (31). The rules first developed and validated for the PT were used with one exception. Namely, the anterior boundary was delineated by tracking from the retroinsular end of Heschl's Sulcus (HS) to the STG. GM voxels closer to the PT overlapping surface were included in the statistical analyses. In order to get a distance map for the GM, the distance between the centroid (center of mass) of each GM voxel and the closest GM/WM surface vertex was calculated, with $1 \mathrm{~mm} \times 1 \mathrm{~mm} \times 1 \mathrm{~mm}$ resolution. These data give information on the probability distribution of the GM distance from the GM/WM surface. The PT surface provides the natural local coordinates associated with the GM/WM surface. The third dimension is described by the normal coordinates measuring the distance of the GM voxel from the surface yielding LCDMs. Figure 1 illustrates the computation of distances for the LCDMs.

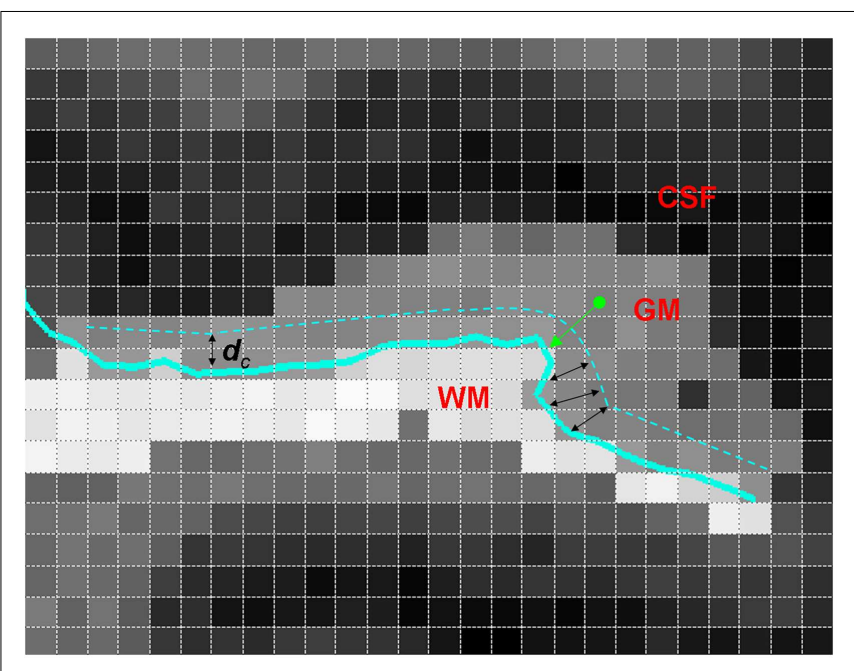

FIGURE 1 | A two-dimensional illustration of LCDM distance computation (i.e., the normal distance) from a GM voxel to the GM/WM PT surface (thick green arrow). Also shown is the censoring procedure with censoring distance $d_{c}$ (black double arrows). At this censoring step, the GM voxels with centroid closer to the GMMM surface than $d_{\mathrm{c}}$ are retained.

\section{STATISTICAL ANALYSIS \\ Pooled LCDM}

Following Ceyhan et al. (27), the LCDM distances were combined across subjects within each diagnostic group, resulting in a set of distances called pooled distances. Distances were restricted to the range of -1 to $4.5 \mathrm{~mm}$ for anatomical reasons (17). This resulted in an average of 0.42 and $0.50 \%$ of distance values being discarded from the left planum temporale (LPT) and RPT data, respectively. The pooled LCDMs were statistically compared using non-parametric tests against the null hypothesis that they come from the same distribution. Kruskal-Wallis (KW) tests were used for the three-group analyses. Any significant results were followedup using Mann-Whitney $U$ (MWU) tests, Wilcoxon Rank Sum tests, and Kolmogorov-Smirnov (KS) tests for pairwise comparisons as post hoc tests, comparing all pairs of diagnostic groups. Each test was initially two-sided. If significant, one-sided (i.e., greater than and less than versions of the) tests were performed to determine the direction of significant difference. Holm's correction was applied to $p$-values to account for the multiple pairwise comparisons between the diagnostic groups (32).

In this method of pooled distances, between-sample independence appears to hold (i.e., pooled LCDM distances between the diagnostic groups can be assumed to be independent); however, within-sample independence is violated as the distances for each individual are not independent. This dependence arises from voxels of the same subject having spatial dependence on nearby voxels. Although pooling does not mitigate this dependence problem, it has been shown that its influence is negligible when employing non-parametric tests based on ranking of the distances (27). Despite this assumption violation, a major advantage of our method is the ability to use all of the information provided by LCDM distances. The fact that the sample sizes are large is for the 


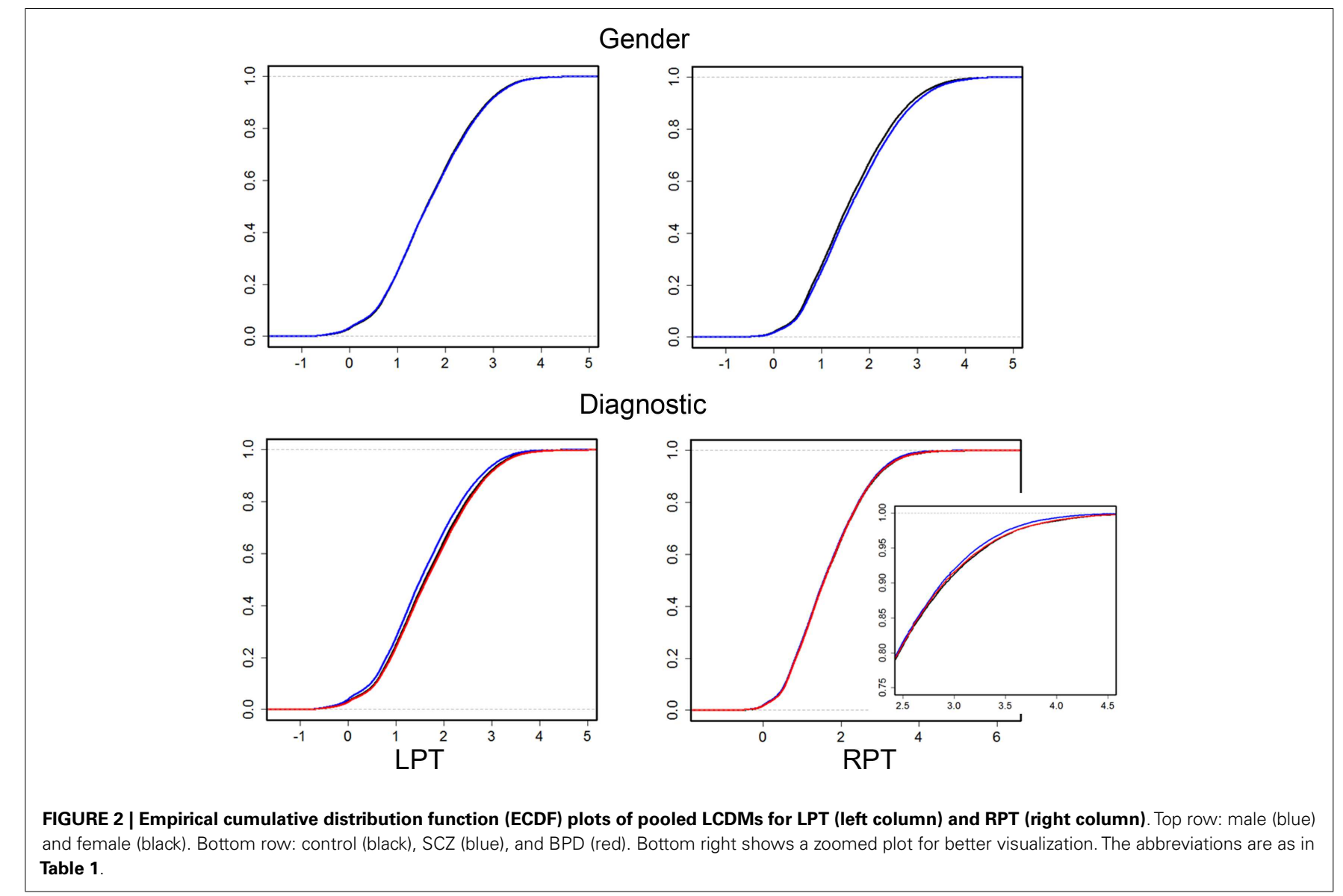

most part not causing spurious significance. Although the pooled distances emphasize the main traits, individual variations in morphometry provide sufficient noise to counterbalance the effects of very large number of data points.

\section{Censored LCDM}

Following Ceyhan et al. (28), the pooled LCDM distances were tested at distances increasing by fixed increments of $0.01 \mathrm{~mm}$. That is, for the first censoring step, pooled distances $\leq 0.01 \mathrm{~mm}$ were tested for group differences; at the second step, distances $\leq 0.02 \mathrm{~mm}$ were tested; and so forth until the maximum distance was reached (where at this last stage, censored distance analysis is equivalent to the pooled distance analysis). Figure 1 illustrates censoring at the threshold distance $d_{c}$ where the GM voxels whose centroids are closer to the GM/WM surface than $d_{\mathrm{c}}$ are retained for analysis at that censoring step. The $p$-values were computed at each censoring step for the KW and MWU tests. Again Holm's correction was applied to $p$-values to account for the pairwise comparisons.

Censored LCDM distances inherit the strengths and weaknesses of the pooled LCDM distances. In particular, the problem of within-sample dependence at each censoring step persists, but this was again shown to be negligible (28). On the other hand, censoring provides a powerful methodology that can identify at which distance values (with respect to the GM/WM surface) the potential differences start to occur. Thus, it provides information that is not available from the pooled distances. One further note is that, by construction, KS tests are not meaningful for censoring distance analysis and therefore were not performed on the censored distances.

\section{RESULTS}

Results were obtained with statistical significance level set at 0.05. Figure 2 shows the empirical cumulative distribution function (ECDF) plots of pooled LCDMs for the LPT and RPT; the small negative distances reflect the sub-voxel inaccuracy of the generated surface. Multi-group comparisons for the two structures revealed overall differences between groups (KW, $p<0.001$ for all except $p=0.0098$ for RPT). Table 1 shows $p$-values for the MWU tests for pairwise comparisons of diagnostic groups and of gender. Table 2 shows $p$-values for KS tests for pairwise comparisons of diagnostic groups. Table 3 shows $p$-values for KS tests for pairwise comparisons of gender. Table 4 shows the results from the MWU tests of censored distances with significant $p$-value cutoffs for LPT and RPT, by diagnosis. Also shown are the results of the KW tests for all groups. Table 5 shows the censored distance analysis for the PT by gender. Figure 3 shows $p$-value plots of censored analysis of the LPT and RPT by diagnosis, based on the MWU test.

\section{DISCUSSION}

This paper describes the application of non-parametric statistical methodology to provide a different and complementary 
Table 1 | Comparison of thickness $(\mathrm{mm})$ in diagnostic groups and gender with pooled LCDM analysis.

\begin{tabular}{lll}
\hline & MWU $\boldsymbol{p}$-value & Conclusion \\
\hline $\begin{array}{c}\text { CON vs. SCZ } \\
\text { LPT }\end{array}$ & $<0.001$ & CON thicker than SCZ \\
RPT & 0.0312 & No significance \\
CON vs. BPD & & \\
LPT & $<0.001$ & CON thicker than BPD \\
RPT & 0.8444 & No significance \\
SCZ vs. BPD & & \\
LPT & $<0.001$ & SCZ thinner than BPD \\
RPT & 0.0208 & No significance \\
M vs. F & & \\
LPT & 0.1762 & No significance \\
RPT & $<0.001$ & Males thicker than females
\end{tabular}

CON stands for controls, SCZ for schizophrenia, BPD for psychotic bipolar disorder, MWU for Mann-Whitney U test, LPT for left planum temporale, RPT for right PT. M and F stand for males and females, respectively.

Table 2 | Kolmogorov-Smirnov (KS) test for ECDF comparisons of pooled LCDM distances in diagnostic groups.

\begin{tabular}{ccccl}
\hline & \multicolumn{3}{c}{ KS test } & Conclusion \\
\cline { 2 - 3 } & Two-sided & $\begin{array}{l}\text { 1st ECDF }< \\
\text { 2nd ECDF }\end{array}$ & $\begin{array}{c}\text { 1st ECDF }> \\
\text { 2nd ECDF }\end{array}$ \\
\hline CON vs. SCZ & & & \\
LPT $\quad<0.001$ & $<0.001$ & 0.9999 & CON thicker than SCZ \\
RPT $\quad 0.1473$ & 0.1715 & 0.9999 & No significance \\
CON vs. BPD & & & \\
LPT $\quad<0.001$ & $<0.001$ & 0.9999 & CON thicker than BPD \\
RPT $\quad 0.6314$ & 0.9999 & 0.9999 & No significance \\
SCZ vs. BPD & & & \\
LPT $\quad<0.001$ & 0.9999 & $<0.001$ & SCZ thinner than BPD \\
RPT & 0.1473 & 0.9999 & 0.2415 & No significance \\
\hline
\end{tabular}

Other abbreviations are as in Table 1.

Table 3 | KS test for ECDF comparisons of pooled LCDM distances in gender groups

M vs. F KS (two-sided) KS (<) KS (>) Conclusion

\begin{tabular}{lllll}
\hline LPT & 0.0022 & 0.0011 & 0.4184 & Males thicker than Females \\
RPT & $<0.001$ & $<0.001$ & 0.9999 & Males thicker than Females
\end{tabular}

Abbreviations are as in Tables $\mathbf{1}$ and $\mathbf{2}$.

perspective of LCDMs with reference to the PT in a differential analysis of SCZ and psychotic BPD in a medium sized population. The three non-parametric statistical tests (i.e., KW, MWU, and KS) were chosen because the distribution of LCDM distances is clearly non-Gaussian. All three tests are sensitive to differences in the distribution of LCDM distances but provide different aspects of the information conveyed by LCDM distances
Table 4 | Censored significant $p$-value cutoffs for LCDM distances compared by diagnosis.

Multi-group comparison by $\mathrm{KW}$ test

\begin{tabular}{lc}
\hline LPT & $(0.35,4.5)$ \\
RPT & - \\
\hline
\end{tabular}

Pairwise comparisons by MWU test

\begin{tabular}{lccc}
\hline Comparison & ROI & \multicolumn{2}{c}{ Direction } \\
\cline { 3 - 4 } & & $\mathbf{1}^{\text {st }}>\mathbf{2}^{\text {nd }}$ & $\mathbf{1}^{\text {st }}<\mathbf{2}^{\text {nd }}$ \\
\hline CON, BPD & LPT & $(1.89,4.5)$ & - \\
SCZ, BPD & RPT & - & - \\
CON, SCZ & LPT & - & $(0.35,4.5)$ \\
& RPT & $(4.35,4.5)$ & - \\
& LPT & $(0.22,4.5)$ & - \\
\hline
\end{tabular}

Numbers listed are the ranges of distances (in millimeters) at which the null hypothesis is rejected. Numbers are in italics when this value is lower than the distance at which the multi-group comparison's null hypothesis is rejected, suggesting that they may not be meaningful below the multi-group comparison significance threshold. ROI stands for region of interest; the other abbreviations are as in Table 1.

Table 5 | Censored significant $\boldsymbol{p}$-value regions for the LCDM distances compared by gender.

\begin{tabular}{lcc}
\hline ROI & \multicolumn{2}{c}{ Direction } \\
\cline { 2 - 3 } & Male $>$ female & Male $<$ female \\
\hline LPT & $(1.35,2.60)$ & - \\
RPT & - & {$[0.88,4.00]$}
\end{tabular}

Results are obtained from the MWU test. Numbers listed are the ranges of thickness (millimeters) in which the null hypothesis is continually rejected. The abbreviations are as in Table 1.

with certain limitations. Conceptually, the KW test is an omnibus test indicating whether distance distributions among two or more groups are different or not, while MWU tests reveal the pairwise group differences. On the other hand, KS tests may provide stochastic ordering (when used together with the corresponding ECDF plots). Given that the KW test shows an overall group difference, the MWU tests yield information about a more biologically meaningful question: "on average, do distances in one group tend to be less than in another group?" A significant MWU test for a left-sided alternative comparing the distances for two groups, say A and B, would imply that a randomly chosen cortical GM voxel tends to be located closer to the GM/WM boundary in group A than in group B (which suggests cortical thinning in group A is more likely). The KS test, like the KW test, tests for differences in the distribution between groups. Unlike the KW test, the KS test can also be adapted to check for stochastic ordering. The usual KS test depends on the largest vertical distance between two ECDFs. The one-sided version of the KS test depends on the largest vertical 

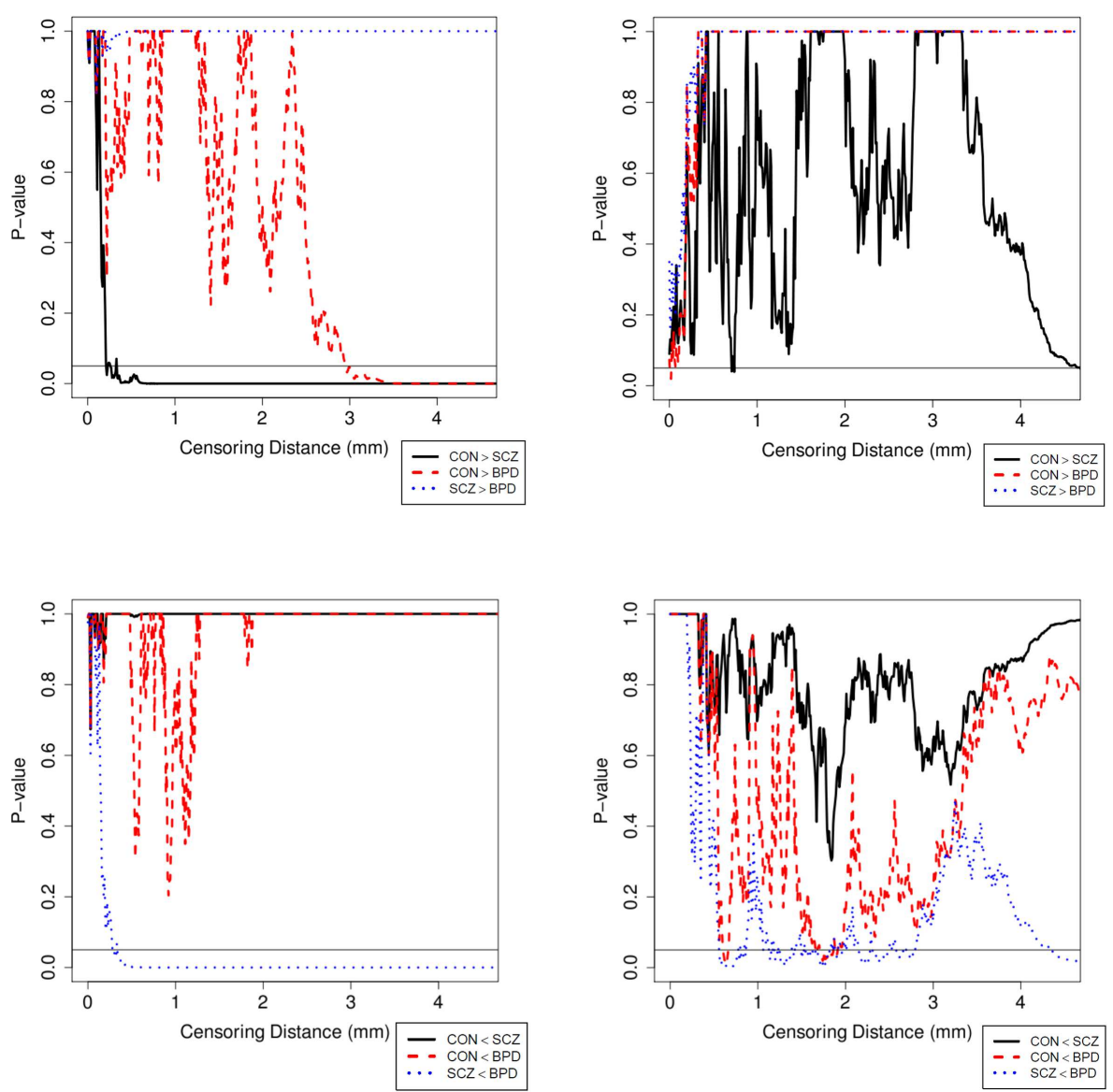

FIGURE 3 | Plot of $\boldsymbol{p}$-values for the MWU test between diagnostic groups in the LPT (left column) and RPT (right column). $p$-Values are adjusted with Holm's correction for multiple comparisons between the diagnostic groups. The abbreviations are as in Table $\mathbf{1}$.

distance between these distributions. Although MWU and KS tests both answer distributional questions, they assess different aspects of the distributional structure. In particular, the MWU test provides differences in the distribution of the ranked distances, while the KS test provides differences in ECDF estimates. Furthermore, the MWU test is complementary, in the sense that it can only be significant for one one-sided alternative and the uncorrected $p$-values for both one-sided alternatives (i.e., left and right side) add up to 1 . In contrast, the $\mathrm{KS}$ test may be significant for both left- and right-sided alternatives, occurring at different distance values. Also, the KS test will almost never be complementary for one-sided tests. Practical and theoretical aspects of pooled and censored LCDM analysis have been discussed extensively elsewhere $(27,28)$. In particular, Monte Carlo simulations suggest that these approaches are capable of capturing differences between diagnostic groups. Furthermore, it is emphasized that censoring should be understood in a pointwise fashion, not as testing a whole range of LCDM distances (28).

Our results confirm that the LPT is more sensitive than the RPT in distinguishing between SCZ, BPD, and healthy controls (Tables 1 and 2; bottom row of Figure 2). Also confirmed is a strong gender effect, with males displaying a thicker PT than females (Table 3; top row of Figure 2). The differences between groups at smaller distances in the LPT suggest that SCZ and BPD have different effects close to the GM/WM surface. This suggests the presence of subtle changes in the cortical mantle (Figure 3). These differences are consistent with those reported elsewhere. For instance, reduced lateralization correlated with increased severity of symptoms, suggesting that laterality is a biological risk for SCZ (33).

At the macroscopic level, pooled and censored analysis of LCDMs may offer a perspective of changes within the cortical mantle. Granted that a flat cortex would result in a top-hat like LCDM profile and a non-uniform distribution of thickness over the cortical region would result in a skewed LCDM profile, it is not surprising that in view of classical differential geometry (34) the LCDM shape is influenced by local curvature or gyrification and thickness. Additionally, the mantle can be viewed as an aggregation of cortical columns whose orientation and spacing contribute to local cortical thickness and curvature (35) and gyrification (6). Thus, macroscopic changes reflected by differences in LCDM shape could be attributed to changes at the microscopic (i.e., cellular) level within these cortical columns. 
At the microscopic level, the cortical mantle comprises six cortical layers that are numbered I-VI as one goes from the outer or pial (i.e., GM/CSF) boundary away from the skull inwards to the GM/WM surface (36). Each layer is thought to comprise different cells such as neuronal, pyramidal, non-pyramidal, and glial cells that are important in neurotransmission between the different layers as well as with other cortical and subcortical regions (36). Estimates of neuronal and glial densities in different cortical regions have been obtained from several histopathological studies in humans and mammals. Altered measures have been suggested as explanations for cortical thinning observed at the macroscopic level in other neuroimaging studies $(37,38)$.

There have been a few histopathological studies of SCZ and BPD in the PT in humans (39-42). Beasley et al. (39) observed reduced neuronal clustering in BPD and SCZ together with reduced neuronal size in layer III of the PT; they suggest that the subtle alterations may be due to neuronal organization within mini-columns or within layers. Smiley et al. $(40,42)$ suggest that reduction in neuron density and volume of layers I-III (deep or infra-granular) may affect differences in LPT in SCZ and that thinner upper layers disrupt multisensory integration and phonetic processing as seen in SCZ. Simper et al. (41) suggested that layer III cell density and pyramidal neuron size differences in the PT are associated with callosal WM abnormality in SCZ, which may affect laterality. Based on these histopathological studies, it is conceivable that differences in LCDMs at distances from the GM/WM surface (Tables 4 and 5; Figure 3) may be characterized by corresponding density changes. For example, reduced density could result in thinner layers and thus altered cortical columns. However, no definitive conclusion can be reached until LCDM analysis can be correlated with measures in histopathological MRI studies of SCZ [e.g., Ref. (38)].

In summary, pooled and censored LCDMs offer alternative and detailed analyses of distances of GM voxels relative to the cortical surface. Further understanding of the biological implications of pooled and censored LCDMs will be necessary. One approach would be to use LDCMs to correlate ante-mortem or post-mortem MRI analyses with histological sections. This would allow for understanding how analysis of LCDM shape is influenced by macroscopic and microscopic changes. Finally, it is affirmed that the specificity of the PT in SCZ suggests that the PT is a contributor to structural abnormalities associated with functional abnormalities in SCZ.

\section{ACKNOWLEDGMENTS}

This work was supported by research grants from the National Institute of Health (R01 MH064838, R01 EB000975, and P41 EB015909) and the EC under the Marie Curie IOF Programme (329370 PRinHDD).

\section{REFERENCES}

1. Panizzon MS, Fennema-Notestine C, Eyler LT, Jernigan TL, Prom-Wormley E, Neale M, et al. Distinct genetic influences on cortical surface area and cortical thickness. Cereb Cortex (2009) 19:2728-35. doi:10.1093/cercor/bhp026

2. Rimol LM, Panizzon MS, Fennema-Notestine C, Eyler LT, Fischl B, Franz CE, et al. Cortical thickness is influenced by regionally specific genetic factors. Biol Psychiatry (2010) 67:493-9. doi:10.1016/j.biopsych.2009.09.032
3. Winkler AM, Kochunov P, Blangero J, Almasy L, Zilles K, Fox PT, et al. Cortical thickness or grey matter volume? The importance of selecting the phenotype for imaging genetics studies. Neuroimage (2010) 53:1135-46. doi:10.1016/ j.neuroimage.2009.12.028

4. Habets P, Marcelis M, Gronenschild E, Drukker M, Van Os J, Genetic R, et al. Reduced cortical thickness as an outcome of differential sensitivity to environmental risks in schizophrenia. Biol Psychiatry (2011) 69:487-94. doi:10.1016/j.biopsych.2010.08.010

5. Joshi AA, Lepore N, Joshi SH, Lee AD, Barysheva M, Stein JL, et al. The contribution of genes to cortical thickness and volume. Neuroreport (2011) 22:101-5. doi:10.1097/WNR.0b013e3283424c84

6. Palaniyappan L, Liddle PF. Differential effects of surface area, gyrification and cortical thickness on voxel based morphometric deficits in schizophrenia. Neuroimage (2012) 60:693-9. doi:10.1016/j.neuroimage.2011.12.058

7. Griffiths TD, Warren JD. The planum temporale as a computational hub. Trends Neurosci (2002) 25:348-53. doi:10.1016/S0166-2236(02)02191-4

8. Hugdahl K, Loberg EM, Nygard M. Left temporal lobe structural and functional abnormality underlying auditory hallucinations in schizophrenia. Front Neurosci (2009) 3:34-45. doi:10.3389/neuro.01.001.2009

9. Ethridge LE, Hamm JP, Shapiro JR, Summerfelt AT, Keedy SK, Stevens MC, et al. Neural activations during auditory oddball processing discriminating schizophrenia and psychotic bipolar disorder. Biol Psychiatry (2012) 72:766-74. doi:10.1016/j.biopsych.2012.03.034

10. Miller MI, Massie AB, Ratnanather JT, Botteron KN, Csernansky JG. Bayesian construction of geometrically based cortical thickness metrics. Neuroimage (2000) 12:676-87. doi:10.1006/nimg.2000.0666

11. Wang L, Hosakere M, Trein JC, Miller A, Ratnanather JT, Barch DM, et al. Abnormalities of cingulate gyrus neuroanatomy in schizophrenia. Schizophr Res (2007) 93:66-78. doi:10.1016/j.schres.2007.02.021

12. Calabrese DR, Wang L, Harms MP, Ratnanather JT, Barch DM, Cloninger CR, et al. Cingulate gyrus neuroanatomy in schizophrenia subjects and their nonpsychotic siblings. Schizophr Res (2008) 104:61-70. doi:10.1016/j.schres.2008. 06.014

13. Harms MP, Wang L, Campanella C, Aldridge K, Moffitt AJ, Kuelper J, et al. Structural abnormalities in gyri of the prefrontal cortex in individuals with schizophrenia and their unaffected siblings. Br J Psychiatry (2010) 196:150-7. doi:10.1192/bjp.bp.109.067314

14. Karnik-Henry MS, Wang L, Barch DM, Harms MP, Campanella C, Csernansky JG. Medial temporal lobe structure and cognition in individuals with schizophrenia and in their non-psychotic siblings. Schizophr Res (2012) 138:128-35. doi:10.1016/j.schres.2012.03.015

15. Qiu A, Younes L, Wang L, Ratnanather JT, Gillepsie SK, Kaplan G, et al. Combining anatomical manifold information via diffeomorphic metric mappings for studying cortical thinning of the cingulate gyrus in schizophrenia. Neuroimage (2007) 37:821-33. doi:10.1016/j.neuroimage.2007.05.007

16. Qiu A, Vaillant M, Barta P, Ratnanather JT, Miller MI. Region-of-interest-based analysis with application of cortical thickness variation of left planum temporale in schizophrenia and psychotic bipolar disorder. Hum Brain Mapp (2008) 29:973-85. doi:10.1002/hbm.20444

17. Chance SA, Tzotzoli PM, Vitelli A, Esiri MM, Crow TJ. The cytoarchitecture of sulcal folding in Heschl's sulcus and the temporal cortex in the normal brain and schizophrenia: lamina thickness and cell density. Neurosci Lett (2004) 367:384-8. doi:10.1016/j.neulet.2004.06.041

18. Ratnanather JT, Poynton CB, Pisano DV, Crocker B, Postell E, Cebron S, et al. Morphometry of superior temporal gyrus and planum temporale in schizophrenia and psychotic bipolar disorder. Schizophr Res (2013) 150:476-83. doi:10.1016/j.schres.2013.08.014

19. Barta P, Miller MI, Qiu A. A stochastic model for studying the laminar structure of cortex from MRI. IEEE Trans Med Imaging (2005) 24:728-42. doi:10.1109/TMI.2005.846861

20. Qiu A, Rosenau B, Greenberg A, Barta P, Yantis S, Miller M. Localizing retinotopic fMRI activation in human primary visual cortex via dynamic programming. Conf Proc IEEE Eng Med Biol Soc (2005) 2:1313-6. doi:10.1109/IEMBS. 2005.1616668

21. Qiu A, Rosenau BJ, Greenberg AS, Hurdal MK, Barta P, Yantis S, et al. Estimating linear cortical magnification in human primary visual cortex via dynamic programming. Neuroimage (2006) 31:125-38. doi:10.1016/j.neuroimage.2005. 11.049 
22. Chuang CH, Cheng PE, Liou M, Liou CW, Kuo YT. Application of SelfOrganizing Map (SOM) for cerebral cortex reconstruction. Int. J. Computational Intelligence Research (2007) 3:32-6. doi:10.5019/j.ijcir.2007.80

23. Chuang CH, Liou JW, Cheng PE, Liou M, Liou CY. Intensity gradient selforganizing map for cerebral cortex reconstruction. Neural Inform Process (2008) 4984:365-73. doi:10.1007/978-3-540-69158-7_39

24. Hutton C, De Vita E, Ashburner J, Deichmann R, Turner R. Voxel-based cortical thickness measurements in MRI. Neuroimage (2008) 40:1701-10. doi:10.1016/j.neuroimage.2008.01.027

25. Gibson E. On the Analysis of Cortical Surface Biomarkers. M.Sc. thesis. Burnaby, BC, Simon Fraser University (2009).

26. Wasserthal C, Brechmann A, Stadler J, Fischl B, Engel K. Localizing the human primary auditory cortex in vivo using structural MRI. Neuroimage (2014) 93:237-51. doi:10.1016/j.neuroimage.2013.07.046

27. Ceyhan E, Hosakere M, Nishino T, Alexopoulos J, Todd RD, Botteron KN, et al. Statistical analysis of cortical morphometrics using pooled distances based on labeled cortical distance maps. J Math Imaging Vis (2011) 40:20-35. doi:10.1007/s10851-010-0240-4

28. Ceyhan E, Nishino T, Alexopoulos J, Todd RD, Botteron KN, Miller MI, et al. Censoring distances based on labeled cortical distance maps in cortical morphometry. Front Brain Imaging Methods (2013) 4:155. doi:10.3389/fneur.2013. 00155

29. Miller MI, Hosakere M, Barker AR, Priebe CE, Lee N, Ratnanather JT, et al. Labeled cortical mantle distance maps of the cingulate quantify differences between dementia of the Alzheimer type and healthy aging. Proc Natl Acad Sci U S A (2003) 100:15172-7. doi:10.1073/pnas.2136624100

30. Takayanagi M, Wentz J, Takayanagi Y, Schretlen DJ, Ceyhan E, Wang L, et al. Reduced anterior cingulate gray matter volume and thickness in subjects with deficit schizophrenia. Schizophr Res (2013) 150:484-90. doi:10.1016/j.schres. 2013.07.036

31. Ratnanather JT, Barta PE, Honeycutt NA, Lee N, Morris HM, Dziorny AC, et al. Dynamic programming generation of boundaries of local coordinatized submanifolds in the neocortex: application to the planum temporale. Neuroimage (2003) 20:359-77. doi:10.1016/S1053-8119(03)00238-6

32. Holm S. A simple sequentially rejective multiple test procedure. Scand J Stat (1979) 6:65-70.

33. Oertel V, Knochel C, Rotarska-Jagiela A, Schonmeyer R, Lindner M, Van De Ven V, et al. Reduced laterality as a trait marker of schizophrenia - evidence from structural and functional neuroimaging. J Neurosci (2010) 30:2289-99. doi:10.1523/JNEUROSCI.4575-09.2010

34. Younes L. Shapes and Diffeomorphisms. Berlin: Springer-Verlag (2010). 438 p. doi:10.1007/978-3-642-12055-8

35. Seldon HL. Cortical laminar thickness and column spacing in human temporal and inferior parietal lobes: intra-individual anatomical relations. Laterality (2006) 11:226-50. doi:10.1080/13576500500489162
36. Jones EG. Cerebral cortex. In: Squire LR, editor. Encylopedia of Neuroscience. Oxford: Academic Press (2004). p. 769-73. doi:10.1016/B978-008045046-9. 02027-1

37. Selemon L. Increased cortical neuronal density in schizophrenia. Am J Psychiatry (2004) 161:1564. doi:10.1176/appi.ajp.161.9.1564

38. Selemon LD, Ceritoglu C, Ratnanather JT, Wang L, Harms MP, Aldridge K, et al. Distinct abnormalities of the primate prefrontal cortex caused by ionizing radiation in early or midgestation. J Comp Neurol (2013) 521:1040-53. doi: $10.1002 / \mathrm{cne} .23217$

39. Beasley CL, Chana G, Honavar M, Landau S, Everall IP, Cotter D. Evidence for altered neuronal organisation within the planum temporale in major psychiatric disorders. Schizophr Res (2005) 73:69-78. doi:10.1016/j.schres.2004.08.011

40. Smiley JF, Rosoklija G, Mancevski B, Mann JJ, Dwork AJ, Javitt DC. Altered volume and hemispheric asymmetry of the superficial cortical layers in the schizophrenia planum temporale. Eur J Neurosci (2009) 30:449-63. doi:10.1111/j. 1460-9568.2009.06838.x

41. Simper R, Walker MA, Black G, Di Rosa E, Crow TJ, Chance SA. The relationship between callosal axons and cortical neurons in the planum temporale: alterations in schizophrenia. Neurosci Res (2011) 71:405-10. doi:10.1016/j.neures.2011.08. 007

42. Smiley JF, Rosoklija G, Mancevski B, Pergolizzi D, Figarsky K, Bleiwas C, et al. Hemispheric comparisons of neuron density in the planum temporale of schizophrenia and nonpsychiatric brains. Psychiatry Res (2011) 192:1-11. doi:10.1016/j.pscychresns.2010.11.007

Conflict of Interest Statement: The authors declare that the research was conducted in the absence of any commercial or financial relationships that could be construed as a potential conflict of interest.

Received: 28 January 2014; accepted: 16 July 2014; published online: 01 August 2014. Citation: Ratnanather JT, Cebron S, Ceyhan E, Postell E, Pisano DV, Poynton CB, Crocker B, Honeycutt NA, Mahon PB and Barta PE (2014) Morphometric differences in planum temporale in schizophrenia and bipolar disorder revealed by statistical analysis of labeled cortical depth maps. Front. Psychiatry 5:94. doi: 10.3389/fpsyt.2014.00094

This article was submitted to Schizophrenia, a section of the journal Frontiers in Psychiatry.

Copyright () 2014 Ratnanather, Cebron, Ceyhan, Postell, Pisano, Poynton, Crocker, Honeycutt, Mahon and Barta. This is an open-access article distributed under the terms of the Creative Commons Attribution License (CC BY). The use, distribution or reproduction in other forums is permitted, provided the original author(s) or licensor are credited and that the original publication in this journal is cited, in accordance with accepted academic practice. No use, distribution or reproduction is permitted which does not comply with these terms. 